\title{
Les pieux maritimes du pont Vasco da Gama
}

\section{WASTIAUX \\ J. DUCROQ \\ F. CORBETTA}

NOVAPONTE, Campenon

Bernard SGE

5, cours Ferdinand-

de-Lesseps

92851 Rueil-Malmaison

Cedex
Les pieux maritimes du pont Vasco da Gama constituaient un de ses enjeux techniques principaux pour le groupement d'entreprises NOVAPONTE (piloté par Campenon Bernard SGE), qui avait pris à son compte les aléas de fondations. Quelques chiffres en témoignent $-8 \mathrm{~km}$ d'estuaire à franchir, soumis à l'action de la marée: - 860 pieux de 1,70 à $2,20 \mathrm{~m}$ de diamètre pour des capacités portantes supérieures à 3000 t sous séisme, fondés jusqu'à - $85 \mathrm{~m}$ sous le niveau moven du fleuve - des cadences de production moyennes de deux pieux battus et un pieu foré par jour.

\section{Maritime piles of Vasco da Gama bridge}

Construction of maritime piles of Vasco da Gama bridge were one of its main technical challenges for the joint venture NOVAPONTE (led by Campenon Bernard SGE), which was carrying the foundation risks. Some figures are self explanatory $-8 \mathrm{~km}$ of estuary to be crossed, under tide effects :

-860 piles, from 1.70 to $2.20 \mathrm{~m}$ diameter, with a bearing capacity of more than $3000 \mathrm{t}$ under earthquake, founded until $-85 \mathrm{~m}$ under water level;

- average rates of construction of two driven piles and one bored pile per day. 


\section{Description générale du projet}

\section{1}

\section{Données générales}

La nouvelle traversée du Tage a été construite afin de faire face au trafic croissant qui amenait le Ponte 25 de Abril à saturation. Elle permet de dévier le trafic autour de Lisbonne, en joignant l'autoroute du Nord A1 en direction de Porto avec la bretelle de Coina et l'autoroute A2 en direction de l'Algarve et de l'Espagne. Elle participe au nouveau développement de la région de Sacavem.

- La traversée fait $17300 \mathrm{~m}$ de long, englobant trois échangeurs, $5 \mathrm{~km}$ sur terre et un viaduc continu de $12300 \mathrm{~m}$, d'une largeur de $30 \mathrm{~m}$.

- Le pont franchit la principale ligne ferroviaire au nord et $9 \mathrm{~km}$ d'estuaire avec trois voies maritimes. Il se termine au sud sur un espace protégé de marais salants.

- La protection de l'environnement comprend la protection contre le bruit, le traitement des eaux de drainage des tabliers dans la zone des marais salants, le contrôle des dragages et un programme de suivi durant la construction.

- Le cas de charge de séisme correspond à une accélération horizontale de $0,45 \mathrm{~g}$, représentant 4,5 fois celle supposée durant l'événement historique du

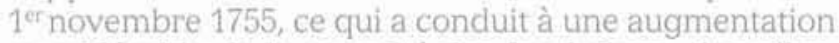
considérable de la capacité des fondations, des piles, des appuis et des joints de dilatation. Différents systèmes antisismiques (amortisseurs, butées latérales) ont été incorporés dans la conception.

\section{2}

\section{Date du projet}

- 1990 : décision de construire la nouvelle traversée et commencement des études préliminaires.

- 1992 : décision de lancer une consultation en concession.

- Janvier 1994 : sélection de deux groupements et commencement des négociations.

- Avril 1994: adjudication du contrat de conception et construction au groupement NOVAPONTE (Campenon Bernard SGE pilote).

- Décembre 1994 : début des installations sur site.

- Août 1995: début des travaux permanents.

- Janvier 1998: fin de la construction.

- 29 mars 1998 : ouverture au trafic.

\section{3}

\section{Les différentes structures (Figs. 1 et 2)} tures :

Le pont de $12300 \mathrm{~m}$ de long est divisé en cinq struc-

- Le viaduc Nord, d'une longueur de 488 m (11 travées d'une moyenne de $45 \mathrm{~m}$ ) est un tablier à nervures multiples en T, d'une hauteur de $3,40 \mathrm{~m}$, d'une largeur variable de 37 à $60 \mathrm{~m}$. Les piles, une par nervure, sont construites chacune sur 4 pieux de $1 \mathrm{~m}$ de diamètre.

- Le viaduc Expo, de $672 \mathrm{~m}$ de long (12 travées de $45 \mathrm{~m}$ à $62 \mathrm{~m}$ ), est un double caisson, de la même hauteur que

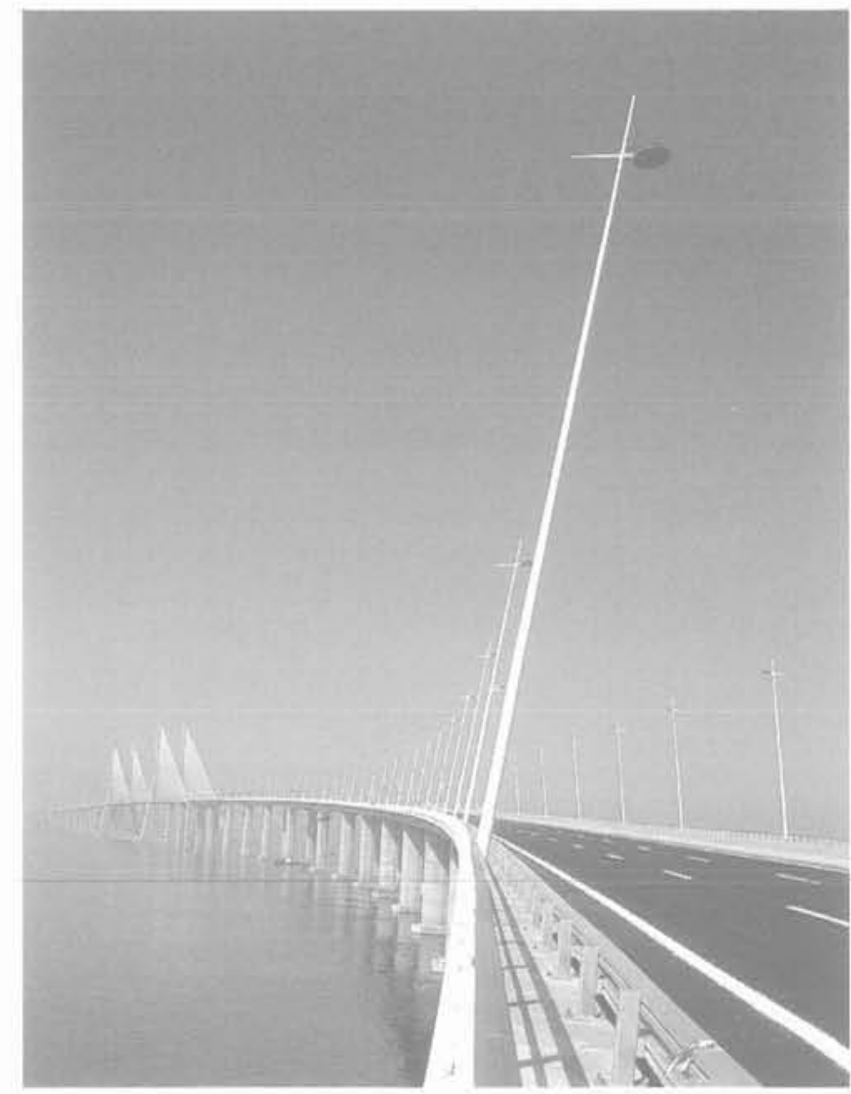

FIG.1 Vue générale depuis le viaduc central avec le pont principal à haubans en fond. General viewtaken from central viaduct towars main cable stay bridge.

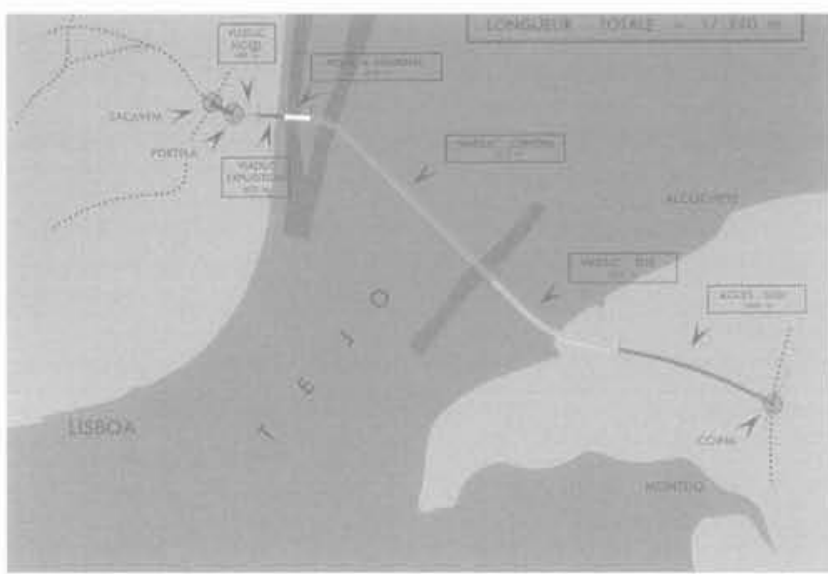

FiG.2 Vue en plan schématique du projet. Schematic plan view of the project.

le viaduc Nord. Les piles comportent deux fûts fondés sur un socle massif ou une semelle avec 12 pieux de $1,70 \mathrm{~m}$ de diamètre.

- Le pont principal, à haubans, fait $820 \mathrm{~m}$ de long, avec une portée centrale de $420 \mathrm{~m}$ au-dessus du principal chenal navigable. C'est un tablier entièrement suspendu (2,60 m de haut) avec deux poutres en béton longitudinales et des poutres transversales en acier tous les $4,40 \mathrm{~m}$. Les $8 \times 24$ haubans sont ancrés sur des pylônes en forme de $\mathrm{H}$, d'une hauteur de $150 \mathrm{~m}$. Le tablier est maintenu transversalement par trois doubles piles de chaque côté de la travée principale. Les fondations sont réalisées en pieux de $2,20 \mathrm{~m}$ (44 par pylône, construits dans un batardeau). 
- Le viaduc central a une lonqueur de $6531 \mathrm{~m}$, en 9 viaducs de 9 travées de $80 \mathrm{~m}$ environ. Chaque travée es faite de 2 caissons préfabriqués de $2000 \mathrm{t}$, de $4 \mathrm{~m}$ de haut, transportés et placés en une seule pièce. Chaque chenal navigable comporte des travées spéciales $(93,5 \mathrm{~m} ; 130 \mathrm{~m} ; 93,5 \mathrm{~m})$ construites en voussoirs, en caisson de hauteur variable. Les piles à doubles füts reposent sur 8 pieux $(1,70 \mathrm{~m}$ pour les travées standards, 2,20 m pour les travées de navigation).

- Le viaduc Sud, de 3825 m de longueur, a une section similaire à celle du viaduc Nord. Le tablier est supporté par des chevêtres, chacun construit sur 4 piles-pieux de $1,80 \mathrm{~m}$ ou $2 \mathrm{~m}$ de diamètre.

Principales quantités

Dragages :

Terrassements généraux :

Pieux $\phi 0,700 \mathrm{~m}$; à 2,200 m:

Bétons :

Surface de tabliers:

Coffrages :

Armatures passives :

Aciers de précontrainte:

Aciers pour haubans :

Effectif maximal de la maitrise :

Effectif maximal des ouvriers:

\section{$3300000 \mathrm{~m}^{3}$ \\ $1570000 \mathrm{~m}^{3}$ \\ 1916 \\ $700000 \mathrm{~m}^{3}$ \\ $400000 \mathrm{~m}^{2}$ \\ $1218000 \mathrm{~m}^{2}$ \\ $100000 \mathrm{t}$ \\ $15000 \mathrm{t}$ \\ $1670 \mathrm{t}$ \\ 350 personnes \\ 3000 personnes}

\section{2}

\section{Données géotechniques}

\section{1}

\section{La campagne géotechnique complémentaire}

Elle s'est déroulée de juillet à novembre 1994, à partir de trois barges travaillant en parallèle. Au droit de chaque pile en rivière une reconnaissance a été faite incluant suivant les cas :

- des pénétromètres statiques (SPT);

- des pénétromètres dynamiques (CPT);

- des carottages et prélèvements d'échantillons intacts:

- des scissomètres dans les vases :

- quelques pressiomètres à titre complémentaire; - des essais dynamiques (còne sismique et crosshole).

Sur les échantillons prélevés, on a pratiqué une gamme complete d'essais en laboratoire :

- analyses granulométriques, chimiques, de densité et de perméabilité ;

- teneurs en eau et limites d'Atterberg ;

- triaxiaux statiques (UU, CU, CD) et cycliques ;

- essai de cisaillement;

- colonne résonnante, torsion cyclique ;

- cedomètres.

\section{2,2}

\section{Profil au droit de l'ouvrage (Fig. 3)}

Le sol de fondation consiste essentiellement en la superposition de deux complexes stratigraphiques:

\section{1) Le complexe alluvial holocénique}

Il comprend les dépôts alluvionnaires existant dans l'estuaire du Tage, d'épaisseur totale $65 \mathrm{~m}$ à $80 \mathrm{~m}$. divisé en quatre couches différentes:

- la couche $\mathrm{a}_{0}$ : vase fine noire avec quelques inclusions de sable et coquillages, d'épaisseur de 30 à $40 \mathrm{~m}$ :

-SPT: de 0 et 4 (dans les 10 premiers mètres),

- Cu : de 6 à $40 \mathrm{kPa}$ (dans les 10 premiers mètres); - la couche a. constituée de sable fin à moyen, gris marron sombre :

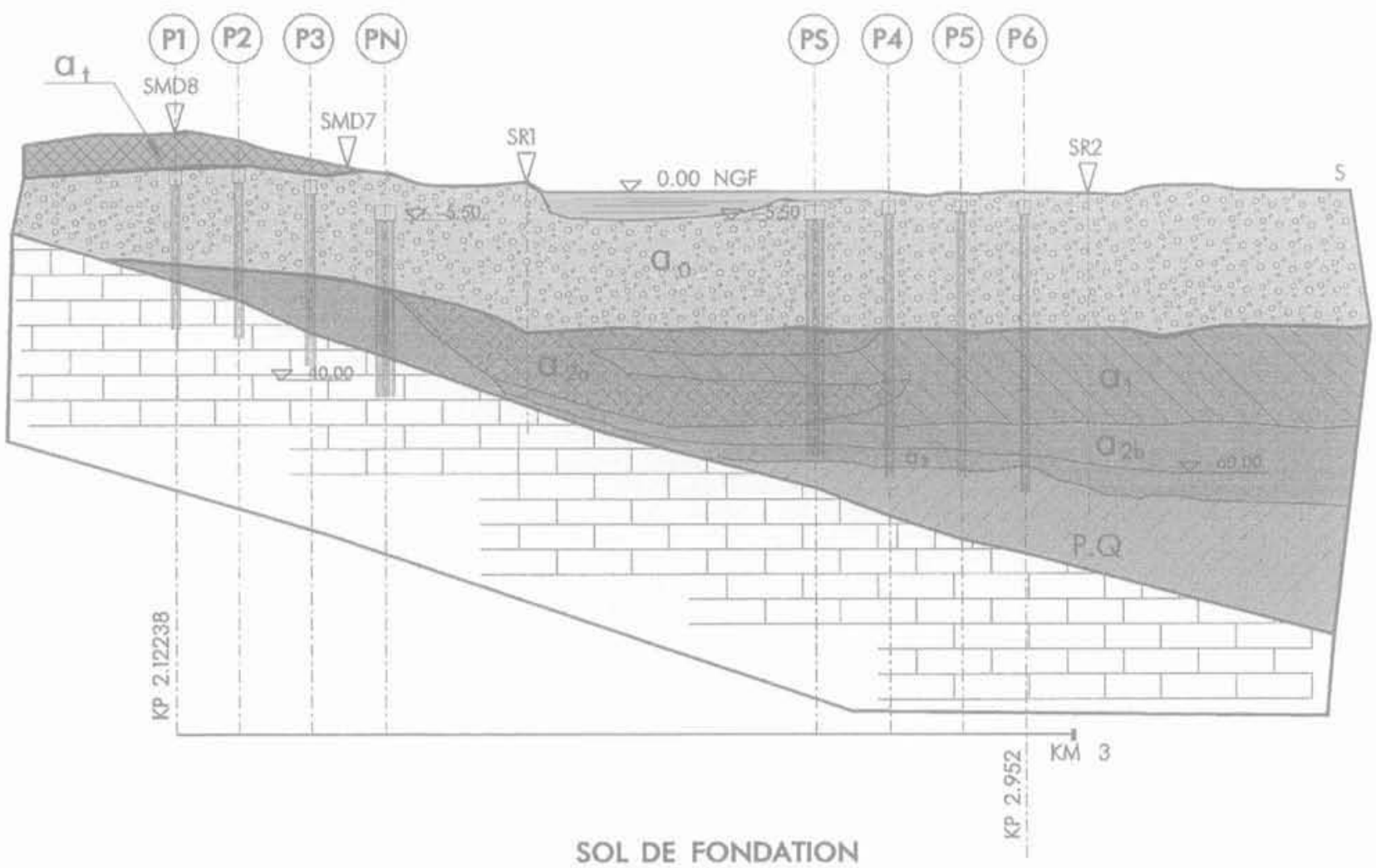

FG. 3 Coupe géotechnique longitudinale au droit du pont principal à haubans. Longitudinal geotechnical cross section at main cable stay bridge. 
- passant au tamis 200: 10 à $20 \%$,

- SPT : de 15 à 60,

- CPT : qc compris entre 3 et $30 \mathrm{MPa}$,

$-\varnothing: 36^{\circ}$

- la couche $\mathrm{a}_{2}$ a d'argile silteuse, beige à gris sombre ;

-SPT de 10 à 45,

- CPT : qc compris entre 1 et $5 \mathrm{MPa}$,

$-\mathrm{Cu}: 100 \mathrm{kPa}$;

- la couche a,b constituée de sable moyen à grossier, beige à gris clair :

- passant au tamis 200: 5 à $15 \%$,

- SPT de 15 à 60,

-CPT: qc compris entre 10 et $35 \mathrm{MPa}$,

$-\varnothing: 40^{\circ}$;

- la couche $a_{3}$ de graviers et galets fins à grossiers.

$-\mathrm{SPT}>60$;

$-\varnothing=42^{\circ}$.

2) Le subtratum pliopléistocène (PQ)

Il comporte des alternances de couches compactes en majorite sableuses (sable fin à grossier) et argileuses (raides à dures) quelquefois cimentées.

La stratification est peu régulière avec un pendage moyen de 5 à $7^{\circ}$ vers l'est :

- SPT de 15 à 60 (argiles) ;

$-\varnothing=40^{\circ}$ (sables).

\section{3}

\section{Critères de définition des pieux maritimes}

\section{1}

\section{Les quantités et les charges}

- Pont principal à haubans :

- Viaduc central
152 pieux de diamètre $2,20 \mathrm{~m}$, charge verticale $\mathrm{Q}=$ $31000 \mathrm{kN}$;

584 pieux de diamètre $1.70 \mathrm{~m}$ charge verticale $\mathrm{Q}=$ $20000 \mathrm{kN}$, à $30000 \mathrm{kN}$, 64 pieux de diamètre $2,20 \mathrm{~m}$,

charge verticale $\mathrm{Q}=$ $60000 \mathrm{kN}$;

- Viaduc Sud (partie maritime) 60 pieux de diamètre $2,20 \mathrm{~m}$.

\section{2}

\section{Les contraintes à respecter}

Les principales contraintes liées au site étaient les suivantes:

- L'étendue du site : l'ensemble des 860 pieux maritimes s'étend sur une longueur de $7900 \mathrm{~m}$.

- La marée : le plan d'eau est soumis aux effets de la marée bidiurne. L'amplitude maximale est de 4,00 m.

- Un clapot d'amplitude maximale $1,20 \mathrm{~m}$ peut intervenir, généré par le vent; ce phénomène est plus particulièrement sensible dans la zone sud du chantier.
- Les courants de marée dont la vitesse en surface peuvent atteindre 3 nœuds dans les passes navigables.

- La bathymétrie du site. La majorité du tracé, hormis les zones des passes navigables, émerge à marée basse de vives eaux. Un chenal d'accès de largeur $200 \mathrm{~m}$, perpendiculaire à l'estuaire, a été dragué de manière à garantir l'accès à toute heure de la marée à des embarcations de deux mètres de tirant d'eau.

- La permanence de la navigation commerciale dans les passes navigables et l'obligation de garantir la permanence de ce trafic. Cette contrainte s'est révélée légère.

- L'environnement maritime du site, la présence de la faune, la gestion des zones de dépòt des matériaux excavés.

- La difficulté relative à identifier et négocier l'utilisation de zones terrestres en bordure d'estuaire permettant la fabrication, le stockage, le chargement ou déchargement des éléments constitutifs des pieux.

Les principales contraintes liées au projet étaient : - La définition des pieux avec des charges importantes imposant l'usage de matériels relativement lourds dans des eaux peu profondes.

- Le programme d'exécution des fondations pieux, batardeaux, caissons, très tendu. Les cadences moyennes des pieux êtaient de : 1 pieu par jour pour le pont à haubans et de 2 pieux par jour pour le viaduc central.

\section{Le choix de la technique de construction des pieux}

\section{$323+1$}

Entre la solution des pieux forés chemisés et celle des pieux en acier battus partiellement remplis de béton, l'argumentaire de choix s'établit cornme suit:

a) Pieux forés chemisés

Les points forts :

- une capacité portante plus élevée au ml ;

- une meilleure durabilité ;

- des machines moins lourdes, donc des équipements plus facilement mobilisables :

- des chemises métalliques moins épaisses, plus faciles à fabriquer et à transporter :

- une technique usuelle au Portugal.

Les points faibles :

- des contraintes beaucoup plus sévères à la réalisation (pour pallier les risques durant le forage et le bétonnage) ;

- une logistique très importante : une dizaine de barges par atelier, dont une centrale à béton flottante de capacité $120 \mathrm{~m}^{3} / \mathrm{h}$ (Figs. 4 et 5 ) ;

- une cadence faible par atelier : 2 jours par pieu :

- la nécessité de recéper les têtes de pieu.

b) Pieux aciers battus, partiellement remplis de béton

Les points forts :

- une cadence élevée (2 pieux par jour) ;

- une méthode simple et fiable (Figs. 6, 7 et 8) ; 


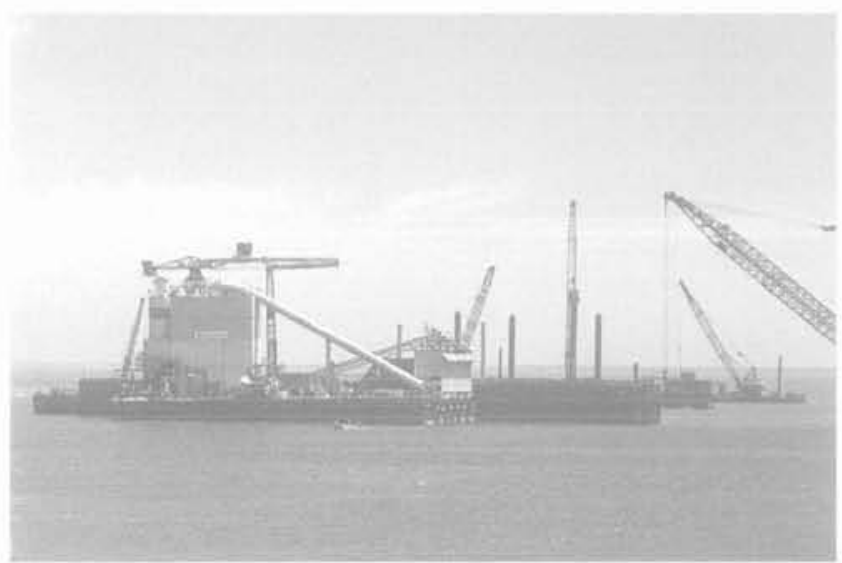

HG. 4 Centrale à béton flottant. Floating batching plant.

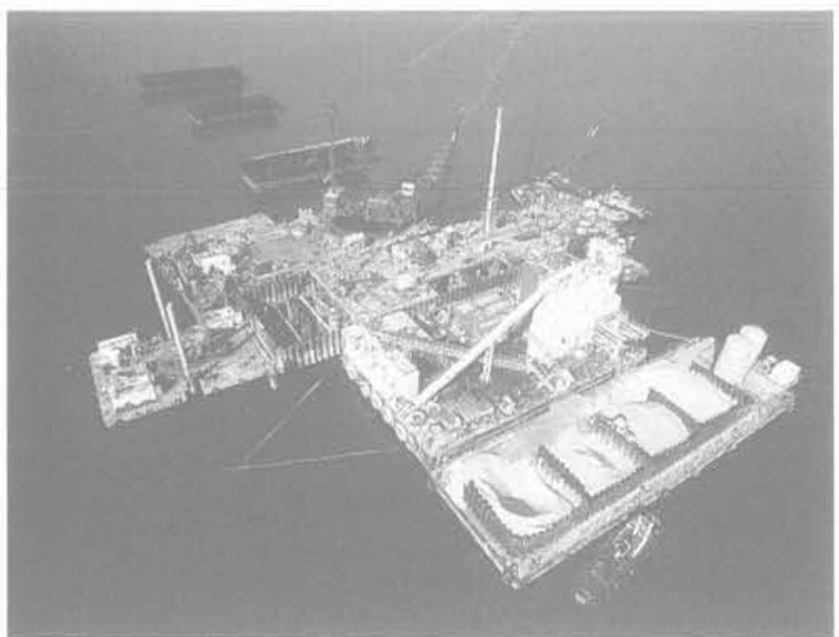

FiG.5 Vue d'ensemble d'un batardeau de pylône. Overall view of a pylon cofferdam.

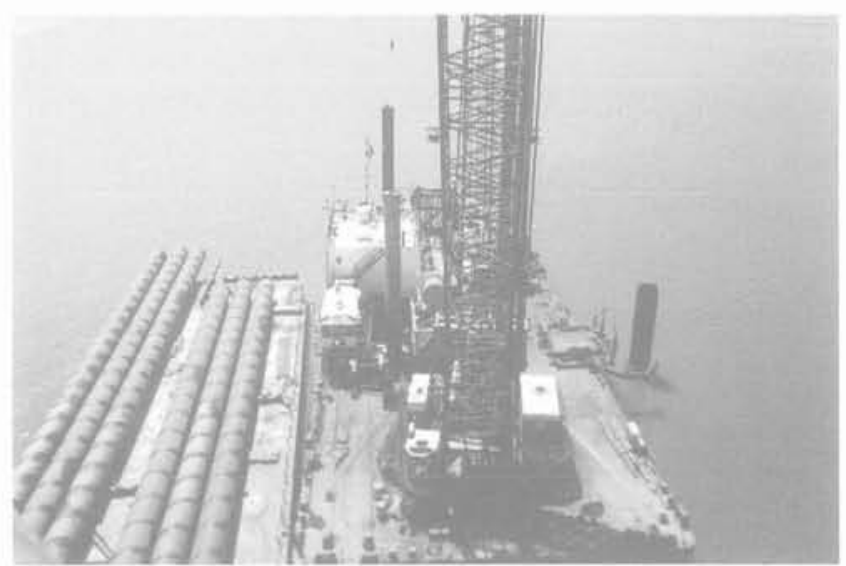

FIG.6 Barge de battage Pile driving barge.

- un atelier de battage plus concentré et mobile.

- la possibilité de contròler la portance des pieux durant le battage et de la corriger facilement si elle est insuffisante :

- la dissociation des opérations de battage, d'excavation (partielle) et de bétonnage, améliorant la flexibilité de la réalisation.

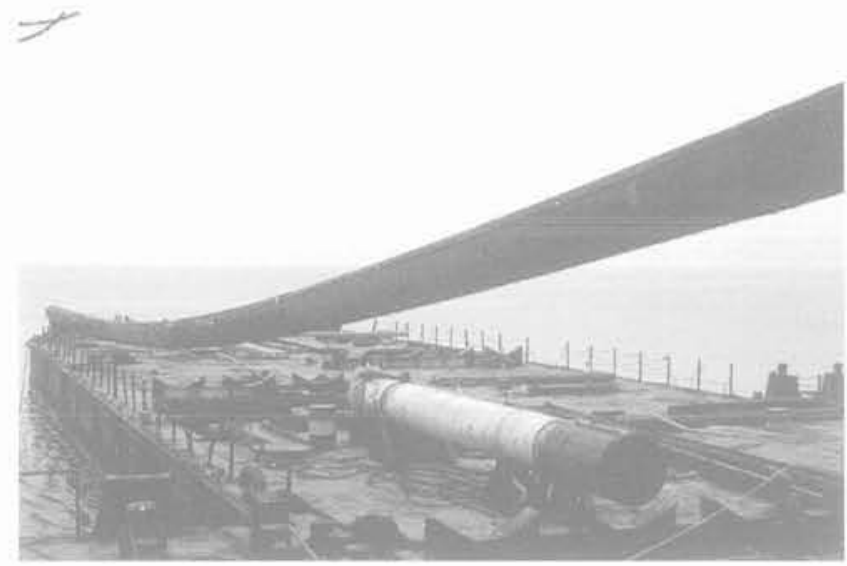

FIG.7 Levage d'un pieu battu. Lifting of a casing for a driven pile.

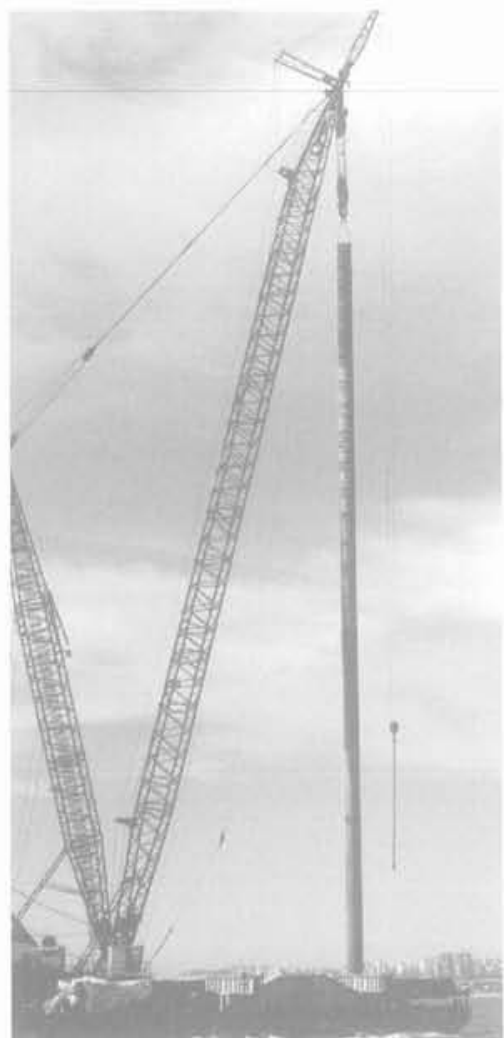

FIG. 8 Mise en fiche d'un pieu battu. Placing of a pile into driving position.

Les points faibles:

- une capacité portante moins bonne au ml;

- un marteau de battage de très forte puissance difficile à remplacer en cas d'avarie (Fig. 9) ;

- la nécessité d'armer la partie supérieure du béton (sur 15 à $17 \mathrm{~m}$ ) pour s'affranchir des problèmes de corrosion (Fig. 10)

- un approvisionnement plus difficile des chemises métalliques. 


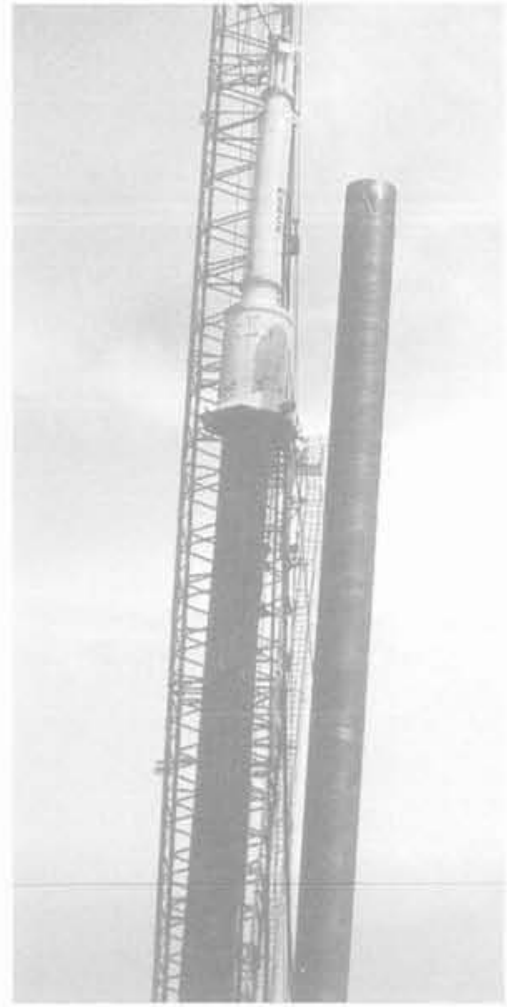

FG. 9 Marteau Menck 500 de $85 \mathrm{t}$. Menck 500 hammer, weight $85 \mathrm{t}$.

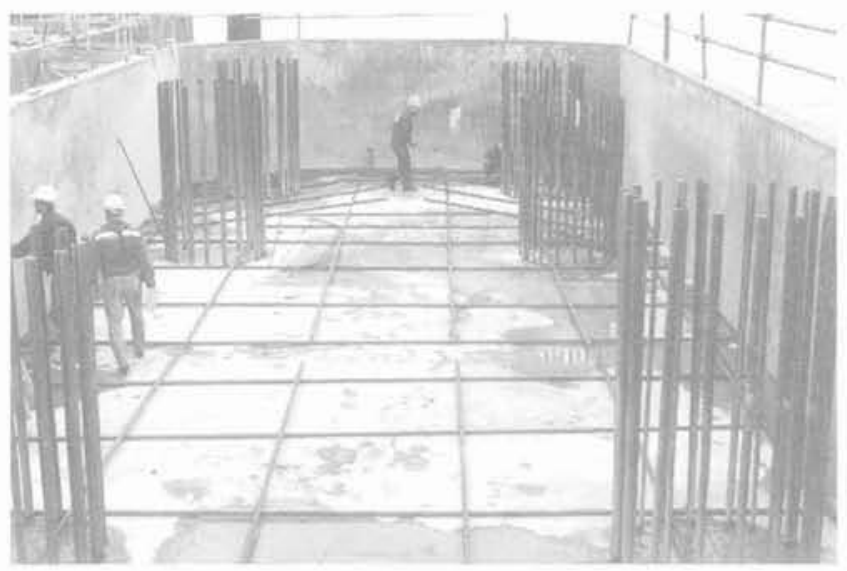

Fig. 10 Vue intérieure d'un caisson de fondation avec la partie supérieure des cages d'armatures des pieux battus.

Inside of a precast foundation caisson with top of pile cages protuding.
- Le viaduc central (parties courantes) :

- site entièrement maritime ;

- grandes cadences;

- on retient les pieux battus.

- Le viaduc central (passes navigables) :

- portance requise très élevée :

- on sélectionne les pieux forés.

- Le viaduc Sud (passes navigables):

- site mixte à majorité terrestre :

- on choisit les pieux forés.

\section{Les essais de pieux (Fig. 11)}

\section{1 \\ Programme des essais}

a) Essais de chargement horizontal. Deux essais ont été réalisés : un pour les pieux battus à $80 \mathrm{~m}$ de la pile 8 (P8) du viaduc central et un pour les pieux forés à $120 \mathrm{~m}$ du pylóne Sud (PS). Le diamètre était de $1,20 \mathrm{~m}$. La charge appliquée a été de $500 \mathrm{kN}$ en P8 et $1000 \mathrm{kN}$ en PS. Ces essais ont permis de déterminer les courbes (p,y) de réaction latérale du sol.

b) Essais de chargement vertical. Au total, neuf essais ont été réalisés sur les différents sites:

- PS (2) : pieux forés;

- P8, P31 (2), P79 (2) P12 (2) : pieux battus. Le diamètre des pieux tests était de $1,20 \mathrm{~m}$ sauf pour P12 $(0,80 \mathrm{~m})$.

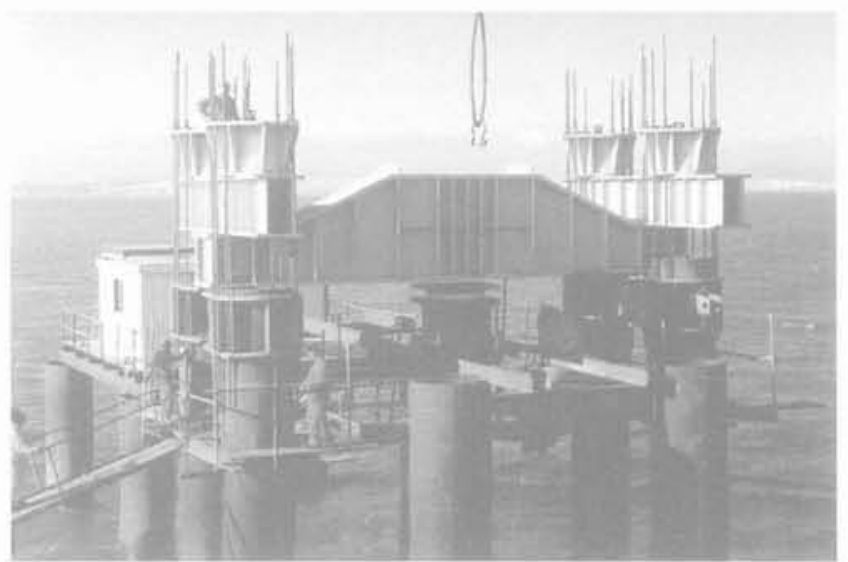

FG 12 Dispositif pour un essai de pieu. Pile test facility.

\section{Les choix retenus résultent d'un compromis pour chaque ouvrage}

- Le pont à haubans:

- site mixte terrestre/maritime :

- pieux concentrés en majorité sous les deux pylônes ; - on choisit les pieux forés. 
- Pieux battus : au contraire des précédents, les paramètres de frottement latéral sont plus faibles que la prévision des formules de Tomlinson. On note l'absence de formation de bouchon. Entre toutes les méthodes envisagées, on a retenu celle du battage du tube sans sabot et de la substitution par du béton sur une partie de la hauteur (jusqu'à $5 \mathrm{~m}$ environ dans les alluvions sous-jacentes à la vase). Cette disposition permet de mobiliser le frottement sur les deux faces de la chemise métallique et a l'avantage d'une grande simplicité d'exécution.

Ces résultats de portance plus faibles que prévu ont obligé à faire pénétrer les pieux les plus chargés dans le substratum pliopléistocénique, pour lequel on disposait de peu d'essais géotechniques. Il a fallu déterminer des critères de battage précis pour pallier ce manque d'information.

\section{5}

\section{Le suivi et les contrôles à la construction}

Le suivi de la construction s'est déroulé dans le cadre du système global de qualité mis en place par NOVAPONTE qui intégrait toute la chaine d'études, de préparation et de production pour les travaux propres et sous-traités conformément à la norme ISO 9001 .

\section{1}

Pieux forés : 276 pieux de diamètre $1,80 \mathrm{~m}, 2 \mathrm{~m}$ et $2,20 \mathrm{~m}$; de longueur maximale $65 \mathrm{~m}$ pour le pont principal à haubans, $78 \mathrm{~m}$ pour les passes navigables.

L'exécution des pieux proprement dits (hors batardeaux) a été sous-traitée à l'entreprise italienne TREVI.

Les contrôles à la construction ont porté sur les paramètres classiques :

- implantation, verticalité, niveau du fond de pieu;

- niveau du fluide de forage, viscosité :

- contrôle des matériaux excavés :

- nettoyage du fond de pieu :

- contrôle de la cage d'armatures:

- niveau et volume de béton mis en place:

- auscultation sonique sur toute la hauteur ( 3 tubes $\phi 50$, 1 tube $\phi 100$ ) ;

- injection de pied à travers les tubes soniques (pression maximale $8 \mathrm{MPa}$ ). Mesure des pressions et débits.

Trois non-conformités ont été enregistrées (dont deux avec effondrement partiel du forage et une avec perte d'outil): Elles ont été résolues par adjonction de pieux supplémentaires, d'une manière compatible avec la géométrie de la fondation concernée.
Les pieux battus : 584 pieux de diamètre $1,70 \mathrm{~m}$, de longueur maxi $85 \mathrm{ml}$.

Les opérations de battage ont été sous-traitées au groupement Volker Stevin-Ballast Nedam. Le positionnement des pieux avec une tolérance absolue de 5 à $10 \mathrm{~cm}$ a été obtenu grâce au GPS différentiel. On s'est ainsi affranchi des conditions de visibilité dars l'estuaire.

Pour les raisons indiquées plus haut, il a été nécessaire de développer une procédure de contrôle de la force portante a partir des résultats du battage. Pour établir cette procédure de contrôle, on s'est appuyé sur des mesures dynamiques faites sur certains pieux par le laboratoire hollandais TNO (en vue de déterminer le rendement énergétique, les coefficients de viscosité des sols) et sur des mesures de refus au battage et au rebattage sur les pieux d'essai et les premiers pieux de l'ouvrage.

La procédure retenue se résume ainsi :

- détermination de la forcé portante Qkd nècessaire pour un pieu, compte tenu des charges appliquées et de certains facteurs parasites comme la liquéfaction sous séisme;

- détermination des résistances statiques nécessaires au battage et au rebattage à 1 jour:

$$
\mathrm{Q}_{\mathrm{B} 0}=\frac{\mathrm{Qkd}}{1,39} \text { et } \mathrm{Q}_{\mathrm{B} 1}=\frac{\mathrm{Qkd}}{1,12}
$$

- calcul par le logiciel WEAP des nombres de coups par mètre nécessaires, respectivement $B_{0}$ et $B_{1}$ :

- vérification du critère de battage : nombre de coups $\mathrm{B}>\mathrm{B}_{0+\text { ? }}$

- si le critère de battage n'est pas vérifié, vérification cu critère de rebattage à 1 jour $(B>B$,

- si ce deuxième critère n'est pas vériffé, reprise cu battage en vue d'approfondir le pieu.

\section{Conclusion}

Les fondations maritimes du Pont Vasco da Gama ont èté riches d'enseignements. Le groupement d'entreprises ayant pris à son compte l'aléa du sol et des systèmes de fondation dans un site alluvionnaire, avec un délai global très tendu, il était nécessaire:

- de prévoir une campagne géotechnique complémen. taire relativement lourde, ainsi qu'un grand nombre d'essais de pieux in situ:

- de choisir um éventail de méthodes de construction éprouvées et permettant de répondre aux problèmes potentiels :

- de se doter de moyens surpuissants en capacité et en cadence, 